OPEN ACCESS

Edited by:

Alfredo G. Torres,

University of Texas Medical Branch,

USA

Reviewed by:

Joerg Graf,

University of Connecticut, USA

Stephen Peter Kidd,

University of Adelaide, Australia

${ }^{*}$ Correspondence:

Yongjie Liu

liuyongjie@njau.edu.cn

Received: 11 July 2016 Accepted: 28 November 2016 Published: 12 December 2016

Citation:

Dong Y, Liu J, Pang M, Du H, Wang N,

Awan F, Lu C and Liu Y (2016)

Catecholamine-Stimulated Growth of Aeromonas hydrophila Requires the TonB2 Energy Transduction System

but Is Independent of the

Amonabactin Siderophore.

Front. Cell. Infect. Microbiol. 6:183.

doi: 10.3389/fcimb.2016.00183

\section{Catecholamine-Stimulated Growth of Aeromonas hydrophila Requires the TonB2 Energy Transduction System but Is Independent of the Amonabactin Siderophore}

\author{
Yuhao Dong ${ }^{1}$, Jin Liu ${ }^{1}$, Maoda Pang ${ }^{1,2}$, Hechao Du ${ }^{1}$, Nannan Wang ${ }^{1}$, Furqan Awan ${ }^{1}$, \\ Chengping $\mathrm{Lu}^{1}$ and Yongjie Liu ${ }^{1 *}$ \\ ${ }^{1}$ Department of Preventive Veterinary, College of Veterinary Medicine, Nanjing Agricultural University, Nanjing, China, ${ }^{2}$ Key \\ Lab of Food Quality and Safety of Jiangsu Province-State Key Laboratory Breeding Base, Institute of Food Safety, Jiangsu \\ Academy of Agricultural Sciences, Nanjing, China
}

The growth-stimulating effects of catecholamine stress hormones have been demonstrated in many pathogens. However, catecholamine-induced growth and its underlying mechanisms remain poorly understood in Aeromonas hydrophila. The present study sought to demonstrate that norepinephrine (NE), epinephrine (Epi), dopamine (Dopa), and L-dopa stimulate the growth of $A$. hydrophila in iron-restricted media containing serum. NE exhibited the strongest growth stimulation, which could be blocked by adrenergic antagonists. Furthermore, it was demonstrated that NE could sequester iron from transferrin, thereby providing a more accessible iron source for utilization by A. hydrophila. The deletion of the amoA gene associated with amonabactin synthesis revealed that the amonabactin siderophore is not required for NE-stimulated growth. However, the deletion of the TonB2 energy transduction system resulted in the loss of growth promotion by NE, indicating that a specific TonB-dependent outer membrane receptor might be involved in the transport of iron from transferrin. Collectively, our data show that catecholamine sensing promotes the growth of $A$. hydrophila in a manner that is dependent on the TonB2 energy transduction system.

Keywords: Aeromonas hydrophila, stress, catecholamine, TonB2 energy transduction system, amonabactin

\section{INTRODUCTION}

Aeromonas hydrophila inhabits various aquatic environments and is responsible for motile Aeromonad septicemia (MAS), leading to large economic losses in the global aquaculture industry (Galindo et al., 2006). This bacterium also causes intestinal and extraintestinal infections in humans and other animals (Parker and Shaw, 2011). As well as being a beneficial symbiont, this bacterium often resides in the host without causing harm. However, once normal host defenses are compromised, A. hydrophila takes advantage of this opportunity to inflict damage to the host (Parker and Shaw, 2011). Thus, the stress status of a host may determine the outcome of an infection.

Stress is unavoidable in the aquaculture environment. The accumulation of Vibrio splendidus increased in juvenile oysters after mechanical stress, such as shaking (Lacoste et al., 2001). The 
exposure of fish to common stressors such as handling increased incidence of disease (Van Weerd and Komen, 1998). Peters et al. (1988) investigated the response of rainbow trout to the simultaneous exposure to stress and A. hydrophila, and found that this bacterium spread to more organs and was in greater number in the stressed fish compared to the unstressed fish. The mechanism of this phenomenon is unknown. One potential explanation is that stress-associated hormones may be released under stress, and these hormones depress the function of the immune system and exert deleterious effects on the immune response of the host (Freestone and Lyte, 2008). In addition, studies have shown that microbes can recognize and respond to neurohormone signals to modulate bacterial growth and virulence-associated gene expression (Burton et al., 2002; Sperandio et al., 2003). Norepinephrine (NE) has been reported to increase motility, chemotaxis, and the production of shiga toxins in Escherichia coli O157:H7 (Lyte et al., 1996; Bansal et al., 2007). Furthermore, NE increased both the cellular cytotoxicity and enterotoxicity of Vibrio parahaemolyticus and up-regulated the transcription of type III secretion system-1 genes (Nakano et al., 2007). Dopamine (Dopa) and epinephrine (Epi) have been found to increase biofilm formation in Streptococcus pneumonia (Sandrini et al., 2014). These observations indicate that stress hormones can regulate the behavior of pathogens. To date, information on the link between stress hormones and A. hydrophila remains limited. Only one study has reported that the treatment of $A$. hydrophila cultures with NE resulted in dramatic increases in bacterial growth (Kinney et al., 1999), but the mechanism underlying this has not been investigated.

It has previously been reported that growth promotion by stress hormones might be attributable to increased iron accessibility in bacterial cells (Freestone et al., 2003). And siderophore synthesis and uptake systems are key elements in the mechanism by which stress hormones induce growth (Burton et al., 2002; Freestone et al., 2003). Iron is an essential element for almost all living bacteria. Because of the low bioavailability of iron in the environment, bacteria have developed specific uptake strategies. A. hydrophila has been shown to possess multiple systems for the sequestration of host iron, including heme-bound iron transport (Maltz et al., 2015), the utilization of enterobactin siderophores produced by enterobacteriaceae (Funahashi et al., 2013), and the secretion of amonabactin (Barghouthi et al., 1989). After the ferri-siderophores and other iron sources specifically bind to outer membrane receptors, an energy generation system for transport is required (Postle and Larsen, 2007). During this process, the transmembrane transporter activity and ATP synthase has been demonstrated to increase significantly in abundance to enhance iron transport and maintain cellular iron homeostasis in A. hydrophila (Yao et al., 2016). Nevertheless, it remains unclear how Aeromonas species keep the iron homeostasis and its regulation under stress conditions.

The majority of our knowledge regarding bacteriacatecholamine interactions originates from studies of mammalian pathogens, and our understanding of the interactions between aquatic bacterial pathogens and catecholamine is relatively lacking. In the current study, we aimed to investigate the impact of $\mathrm{NE}$ and its related compounds on the growth of $A$. hydrophila NJ-35, an isolate from a diseased cyprinoid fish, in iron-restricted media, and to determine whether amonabactin is required for NE-mediated A. hydrophila growth promotion or whether other NE response mechanisms are present.

\section{MATERIALS AND METHODS Ethics Statement}

Experiments involving live animals were carried out according to animal welfare standards and were approved by the Ethical Committee for Animal Experiments of Nanjing Agricultural University, China. All animal experiments complied with the guidelines of the Animal Welfare Council of China.

\section{Bacterial Strains and Growth Media}

The bacterial strains and plasmids used in this study are listed in Table 1. A. hydrophila NJ-35, which belongs to the ST251 clonal group, was isolated from dead cultured cyprinoid fish in the Jiangsu province of China in 2010 (Pang et al., 2015).

TABLE 1 | Bacterial strains and plasmids used in this study.

\begin{tabular}{|c|c|c|}
\hline $\begin{array}{l}\text { Strain or } \\
\text { plasmid }\end{array}$ & Description $^{a}$ & $\begin{array}{l}\text { Source or } \\
\text { reference }\end{array}$ \\
\hline \multicolumn{3}{|l|}{ STRAINS } \\
\hline NJ-35 & $\begin{array}{l}\text { Wilde-type, isolated from diseased } \\
\text { crucian carp, in China }\end{array}$ & $\begin{array}{l}\text { Collected in our } \\
\text { laboratory }\end{array}$ \\
\hline SM10 & E. coli strain, $\lambda$ pir ${ }^{+}, \operatorname{Kan}^{r}$ & $\begin{array}{l}\text { Melton-Witt et al., } \\
2012\end{array}$ \\
\hline$\triangle a m o A$ & amoA deletion mutant from NJ-35 & This study \\
\hline$\Delta \operatorname{ton} B 1$ & tonB1 deletion mutant from NJ-35 & This study \\
\hline$\Delta \operatorname{ton} B 2-1$ & tonB2-1 deletion mutant from NJ-35 & This study \\
\hline$\Delta$ tonB2-2 & tonB2-2 deletion mutant from $\mathrm{NJ}-35$ & This study \\
\hline$\Delta$ tonB3 & tonB3 deletion mutant from NJ-35 & This study \\
\hline \multicolumn{3}{|l|}{ PLASMID } \\
\hline pYAK1 & R6K-ori suicide vector, $\mathrm{SacB}^{+}, \mathrm{Cm}^{r}$ & Abolghait, 2013 \\
\hline pYAK1-amoA & $\begin{array}{l}\text { pYAK1 carrying the flanking sequence } \\
\text { of } a m o A, \mathrm{Cm}^{r}\end{array}$ & This study \\
\hline pYAK1-tonB1 & $\begin{array}{l}\text { pYAK1 carrying the flanking sequence } \\
\text { of tonB1, } \mathrm{Cm}^{r}\end{array}$ & This study \\
\hline pYAK1-tonB2-1 & $\begin{array}{l}\text { pYAK1 carrying the flanking sequence } \\
\text { of tonB2-1, } \mathrm{Cm}^{r}\end{array}$ & This study \\
\hline pYAK1-tonB2-2 & $\begin{array}{l}\text { pYAK1 carrying the flanking sequence } \\
\text { of tonB2-2, } \mathrm{Cm}^{r}\end{array}$ & This study \\
\hline pYAK1-tonB3 & $\begin{array}{l}\text { pYAK1 carrying the flanking sequence } \\
\text { of tonB3, } \mathrm{Cm}^{r}\end{array}$ & This study \\
\hline pMMB207 & Low-copy-number vector, $\mathrm{Cm}^{r}$ & $\begin{array}{l}\text { Morales et al., } \\
1991\end{array}$ \\
\hline pMMB-amoA & $\begin{array}{l}\text { Plasmid pMMB207 carrying the } \\
\text { complete ORF of amoA }\end{array}$ & This study \\
\hline pMMB-tonB2-1 & $\begin{array}{l}\text { Plasmid pMMB207 carrying the } \\
\text { complete ORF of tonB2-1 }\end{array}$ & This study \\
\hline pMMB-tonB2-2 & $\begin{array}{l}\text { Plasmid pMMB207 carrying the } \\
\text { complete ORF of tonB2-2 }\end{array}$ & This study \\
\hline
\end{tabular}

${ }^{a} \mathrm{Cm}^{r}$, chloramphenicol resistant; Kan' ${ }^{r}$ kanamycin resistant. 
A. hydrophila and E. coli strains were grown at 28 and $37^{\circ} \mathrm{C}$, respectively, in Luria-Bertani broth (LB) or on LB agar plates. As necessary, antibiotics were used at the following concentrations: chloramphenicol (Cm) (Sigma, St. Louis, USA), $34 \mu \mathrm{g} / \mathrm{ml}$ for A. hydrophila; ampicillin (Amp) (Sigma), $100 \mu \mathrm{g} / \mathrm{ml}$ for E. coli. Serum-SAPI medium was used to assay growth promotion (Kinney et al., 2000), with some modifications. Briefly, serum-SAPI medium containing $6.25 \mathrm{mM}$ ammonium nitrate, $3.35 \mathrm{mM}$ potassium chloride $2.77 \mathrm{mM}$ dextrose, 1.84 $\mathrm{mM}$ monobasic potassium phosphate and $1.01 \mathrm{mM}$ magnesium sulfate was adjusted to $\mathrm{pH} 7.4$ and supplemented with $10 \mathrm{mM}$ HEPES buffer and $10 \%$ fetal bovine serum. The apo-form of bovine transferrin (ATF), the holo-form of bovine transferrin (HTF), chlorpromazine, phentolamine hydrochloride, and propranolol were purchased from Sigma-Aldrich (St. Louis, MO, USA). Norepinephrine (bitartrate salt), epinephrine (bitartrate salt), dopamine hydrochloride, L-dopa, tyrosine, and tyramine were purchased from Aladdin (Shanghai, China). All reagents were freshly prepared before each experiment and filter-sterilized using $0.22-\mu \mathrm{m}$ (pore-size) membrane filters.

\section{Growth Assays}

For bacterial growth assays, A. hydrophila NJ-35 was grown overnight in $\mathrm{LB}$ medium at $28^{\circ} \mathrm{C}$. Cells were pelleted by centrifugation at $5000 \times \mathrm{g}$ for $10 \mathrm{~min}$, washed and resuspended in phosphate-buffered saline (PBS). An initial inoculum of $\sim 10^{2}$ colony-forming units (CFU) $\mathrm{ml}^{-1}$ was diluted into serum-SAPI medium to obtain the strongest possible growth stimulation. In our pre-experiment, catecholamine hormones were confirmed to have no effect on $A$. hydrophila growth in serum-SAPI medium when the initial inoculum densities were $>10^{3} \mathrm{CFU}$ $\mathrm{ml}^{-1}$ (data not shown). Individual hormones were employed for experimental cultures at a final concentration of $100 \mu \mathrm{M}$, which was selected based on a preliminary study (Figure S1, take NE as an example). Additionally, a catecholamine receptor antagonist at a concentration of $400 \mathrm{mM}$ was added to detect whether it exerted effects on catecholamine-induced growth (Freestone et al., 2007).

To investigate the role of transferrin in NE-induced growth, the serum in SAPI medium was replaced with either $39 \mu \mathrm{M}$ $(3 \mathrm{mg} / \mathrm{ml})$ ATF or HTF. This concentration was chosen on the basis of previous reports that transferrin concentration in mammal serum is in the range $1-3.6 \mathrm{mg} / \mathrm{ml}$ (Burton et al., 2002; Kasvosve and Delanghe, 2002) and our preliminary doseresponse studies, indicating that $3 \mathrm{mg} / \mathrm{ml}$ is the minimum concentration of the transferrin for the optimum growth of A. hydrophila (Figure S2). The initial concentration of bacteria was $1 \times 10^{3} \mathrm{CFU} / \mathrm{ml}$, higher than the concentration used in serum-SAPI medium, since there was no growth of $A$. hydrophila observed with the inoculums densities of $<1 \times 10^{3} \mathrm{CFU} / \mathrm{ml}$. The cultures were grown in $1.5-\mathrm{ml} \mathrm{EP}$ tubes at $28^{\circ} \mathrm{C}$ for $20 \mathrm{~h}$, and $100-\mu \mathrm{l}$ aliquots of bacteria were removed from each tube every $2 \mathrm{~h}$ and plated on LB and the specific growth rate was calculated described previously (Lindqvist and Barmark, 2014). Each experiment was repeated four times.

\section{Biofilm Assay}

A biofilm formation assay were performed utilizing the crystal violet staining method described previously (Stepanovic et al., 2000), with some modification. A. hydrophila NJ-35 was grown overnight in $\mathrm{LB}$ broth at $28^{\circ} \mathrm{C}$, bacteria were washed three times with PBS and diluted to an optical density of 0.01 at 600 $\mathrm{nm}\left(\mathrm{OD}_{600}\right)$ in serum-containing SAPI medium supplemented with $100 \mu \mathrm{M}$ individual hormones, and 200- $\mu \mathrm{l}$ aliquots of suspension were dispensed into 96 -well polystyrene plates. Then, the plates were incubated for $18 \mathrm{~h}$ at $28^{\circ} \mathrm{C}$ without agitation. Next, the contents of the wells were poured off, and wells were washed three times with $300 \mu \mathrm{l}$ of sterile PBS. Adherent bacterial cells were fixed with $200 \mu \mathrm{l}$ of methanol for $15 \mathrm{~min}$, and then the methanol was removed. After drying for $15 \mathrm{~min}$, $200 \mu \mathrm{l}$ of crystal violet $(1 \% \mathrm{wt} / \mathrm{vol})$ per well was added, and staining was carried out for $10 \mathrm{~min}$. Then, the wells were washed with $\mathrm{ddH}_{2} \mathrm{O}$ five times to remove unbound dye, and the plates were air-dried. The formed biofilms were solubilized in absolute ethanol, and the optical density was measured at $\mathrm{OD}_{595}$. Wells that did not contain bacteria served as a negative control.

\section{In vitro Adhesion Assays}

HEp-2 cells were grown in Dulbecco's modified Eagle's medium (DMEM; Gibco, New York, USA) containing 10\% fetal bovine serum (FBS; Gibco, New York, USA) in 24-well tissue culture plates to a final density of $5 \times 10^{5}$ cells/well. The monolayers were washed with sterile $10 \mathrm{mM}$ PBS to remove unattached cells, and then $400 \mu \mathrm{l}$ of MEM without phenol red was added. A. hydrophila NJ-35 was grown in serum-SAPI medium in the presence or absence of $100 \mu \mathrm{M}$ hormones for $18 \mathrm{~h}$ at $28^{\circ} \mathrm{C}$ under a microaerobic atmosphere, then harvested by centrifugation at $6000 \times \mathrm{g}$ for $10 \mathrm{~min}$. The pellet was washed three times with PBS to remove hormones. The bacteria were seeded into each well at a multiplicity of infection (MOI) of $1: 1$. The plates were centrifuged at $600 \times \mathrm{g}$ for $10 \mathrm{~min}$ and incubated in a $5 \% \mathrm{CO}_{2}$ humidified incubator for $2 \mathrm{~h}$ at $37^{\circ} \mathrm{C}$. Subsequently, the cells were washed five times with PBS and lysed by adding $0.02 \%$ Triton X-100. Bacterial numbers were enumerated via serial dilution and plating on LB agar plates (Tenenbaum et al., 2005). All assays were performed with four replicates.

\section{Experimental Infection of Mice}

Six- to eight-week-old female ICR mice were purchased from the Experimental Animal Center of Yangzhou University and housed under specific-pathogen-free conditions. Mice were fed intragastrically with $1 \mathrm{mg}$ of NE in $400 \mu \mathrm{l}$ of PBS $12 \mathrm{~h}$ before bacterial challenge. Mice administered PBS intragastrically served as controls. The dose of NE used in this study was chosen on the basis of a preliminary study, in which the starting dose was screened from the fixed levels of $0.5,1$, and $2 \mathrm{mg}$ expected to show the altered bacterial loads but no obvious clinical signs or pathologic changes in animals. Then, the mice were infected with a predetermined dose of $1 \times 10^{8} \mathrm{CFU}$ of $A$. hydrophila NJ-35 per animal in a $200-\mu l$ suspension by intragastric administration. At $6 \mathrm{~h}$ postinfection, mice were euthanized. Lungs and spleens were 
aseptically removed, homogenized, and diluted in PBS. Bacterial loads in tissues were counted via serial dilution of the suspensions and plating on LB agar plates.

\section{Effects of NE on Iron Release from Transferrin}

To test the ability of NE to acquire iron from Tf, $13 \mu \mathrm{M}(1 \mathrm{mg} / \mathrm{ml})$ HTF was prepared in SAPI medium buffer supplemented with $100 \mathrm{mM}$ Tris- $\mathrm{HCl}$ buffer at $\mathrm{pH} 7.5$, and experimental cultures were supplemented with $100 \mu \mathrm{M} \mathrm{NE}$ and incubated at $37^{\circ} \mathrm{C}$ for $12 \mathrm{~h}$, while control cultures contained an equivalent volume of water only. Samples were analyzed by electrophoresis in $6 \%$ polyacrylamide gels containing $6 \mathrm{M}$ urea in a BioRad Protean II vertical mini gel system as previously described with certain modifications (Wolz et al., 1994). The gels were prepared as follows: $4.5 \mathrm{~g}$ of urea was added into $2.7 \mathrm{ml}$ of acrylamide $(3.3 \%$ C-30\% T) and $2.5 \mathrm{ml} \mathrm{TBE}$ buffer concentrated 5 times, and the volume was adjusted to a total of $12.5 \mathrm{ml}$ with deionized water. The gels were polymerized with the addition of $100 \mu \mathrm{l}$ of $10 \%$ ammonium persulfate and $6 \mu \mathrm{l}$ of TEMED. Electrophoresis was performed at $200 \mathrm{~V}$ for $6 \mathrm{~h}$. The gels were stained with Coomassie Brilliant Blue R-250 (Bio-Rad).

\section{Construction of Gene Deletion Mutants}

$a m o A$ gene inactivation was carried out via homologous recombination using the suicide plasmid pYAK1 as previously described (Abolghait, 2013). First, the left and right arms of the amoA gene were PCR-amplified from the chromosomal DNA of $A$. hydrophila NJ-35 using two sets of primer pairs, AmoA1/AmoA-2 and AmoA-3/AmoA-4 (Table 2), and the arms were then used as templates to generate fusion fragments with the primer pair AmoA-1/AmoA-4. These fusion fragments were cloned into the pYAK1 suicide plasmid with the restriction enzyme BamHI. The recombinant plasmid pYAK1::amoA was transformed into E. coli SM10 competent cells (Melton-Witt et al., 2012). The donor strain E. coli SM10-pYAK1 (Cm resistant, $\mathrm{Cm}^{\mathrm{r}}$ ) and the recipient strain A. hydrophila NJ-35 (Amp resistant, $A m p^{r}$ ) were cultured in LB broth without antibiotics until log phase was reached. Cells were mixed at a ratio of two-to-one vol/vol in medium, spotted on a nylon filter on an LB plate and conjugated for $12 \mathrm{~h}$ at $28^{\circ} \mathrm{C}$. Cells were recovered and washed three times with PBS. LB agar plates containing $100 \mu \mathrm{g} / \mathrm{ml} \mathrm{Amp} \mathrm{and} 34 \mu \mathrm{g} / \mathrm{ml} \mathrm{Cm}$ were used to select for recombinant plasmid integration into the chromosome. Then, colonies $\left(\mathrm{Amp}^{\mathrm{r}}\right.$ and $\mathrm{Cm}^{\mathrm{r}}$ ) were chosen and inoculated in LB broth supplemented with $20 \%$ sucrose to

TABLE 2 | Primers used in this study.

\begin{tabular}{|c|c|c|}
\hline Primer & Sequence $\left(5^{\prime} \rightarrow 3^{\prime}\right)^{\mathrm{a}}$ & Function \\
\hline AmoA-1 & CAGGTCGACTCTAGAGGATCC CTTCGTTCTTGCGAC & Construction of amoA deletion mutant \\
\hline AmoA-2 & ACTGGCTCAT GTTACACCCTCAAATATGATTC & \\
\hline AmoA-4 & GAGCTCGGTACCCGGGGATCC GGCAATCAGCGGGAAACA & \\
\hline TonB1-1 & CAGGTCGACTCTAGAGGATCCGCCTCTGTCTGGTTा & Construction of tonB1 deletion mutant \\
\hline TonB1-4 & GAGCTCGGTACCCGGGGATCCGCACGAACGGGTTATT & \\
\hline TonB2-1-1 & CAGGTCGACTCTAGAGGATCC GTTTCATCTGTCCCTT & Construction of tonB2-1 deletion mutant \\
\hline TonB2-1-2 & ACCGAAATGA CTATGTTGCGGATCTGGA & \\
\hline TonB2-1-3 & CGCAACATAG TCATTTCGGTGCCACC & \\
\hline TonB2-1-4 & GAGCTCGGTACCCGGGGATCC GCGGCTGCTCTACCTCAA & \\
\hline TonB2-2-4 & GAGCTCGGTACCCGGGGATCC AGCCTACAACCGCTACAT & \\
\hline TonB3-1 & CAGGTCGACTCTAGAGGATCC ATGGGATTGCCCTTG & Construction of tonB3 deletion mutant \\
\hline TonB3-2 & GACTATTACAATGAAAGGAATCAAACTTGC & \\
\hline TonB3-3 & TTCCTTCAT TGTAATAGTCCTTGTITCATAG & \\
\hline TonB3-4 & GAGCTCGGTACCCGGGGATCC CCAGACCCAGTTCTATCAG & \\
\hline AmoA-C-F & CAGGAAACAGAATTCGAGCTCTCAGCTGCTCTTGCTCG & Construction of amoA complemented strain \\
\hline AmoA-C-R & GGATCCCCGGGTACCGAGCTCTATCGCCTCCCAGACCA & \\
\hline TonB2-1-C-F & CAGGAAACAGAATTCGAGCTCTTATGACTCCAGTTGAATTGA & Construction of tonB2-1 complemented strain \\
\hline TonB2-1-C-R & GGATCCCCGGGTACCGAGCTCGCAGGCCTATCAAATCGA & \\
\hline TonB2-2-C-F & CAGGAAACAGAATTCGAGCTCTTACGGCTCCGGCTGG & Construction of tonB2-2 complemented strain \\
\hline TonB2-2-C-R & GGATCCCCGGGTACCGAGCTCCGCCGCCAGCCAAC & \\
\hline
\end{tabular}

aUnderlined sequences indicate restriction sites. 
induce a second crossover event (Abolghait, 2013). The doublecrossover $\triangle a m o A$ mutant strain was confirmed by sequencing the deleted region and flanking DNA in the mutated strains. Further, quantitative real-time reverse transcription-PCR (qRTPCR) was used to measure the transcriptional levels of upstream or downstream genes of the deletion regions, demonstrating that no polar mutation occurred due to the knock-out of amoA. Using the same approach, additional deletion mutants including $\Delta$ ton $B 1, \Delta$ tonB2-1, $\Delta$ tonB2-2, and $\Delta$ tonB3 were also constructed.

\section{Construction of the Complementation Strain}

The complementation of $\triangle a m o A$ strain was constructed with shuttle plasmid pMMB207. The DNA fragments, including $a m o A$ gene and its putative promoter and terminator region, were amplified using the primer pair (AmoA-C-F/R) with restriction enzyme sites Sac I. Following digested and purified, the target gene was ligated into the pMMB207 vector. The recombinant plasmid pMMB207-amoA was first introduced into $E$. coli $\mathrm{SM} 10$ by chemical transformation, and then transformed into the mutant strain $\triangle a m o A$ using bacterial conjugation, thus generating the complemented strain $\mathrm{C} \triangle a m o A$. PCR amplification and sequencing were performed to verify the complementation strain. Using the same approach, the complemented strain $\mathrm{C} \Delta$ tonB2-1 and $\mathrm{C} \Delta$ tonB2-2 were also constructed.

\section{Siderophore CAS Plate Assays}

Siderophore production was examined using chrome azurol S (CAS) plate (Schwyn and Neilands, 1987). Wild-type and $\triangle a m o A$ mutant strains were incubated in LB medium overnight, and the cells were collected by centrifugation at $5000 \times \mathrm{g}$ for $10 \mathrm{~min}$, then washed and resuspended in PBS. Cells were normalized to an $\mathrm{OD}_{600}$ of 1 in PBS. Ten microliters of suspension was spotted on a CAS agar plate. The CAS plates were incubated for $24 \mathrm{~h}$ at $28^{\circ} \mathrm{C}$.

\section{Statistical Analysis}

Statistical analyses were performed using SPSS software (SPSS for Windows 16, SPSS Inc., Chicago, IL, USA). Multiple comparisons were performed using analysis of variance (ANOVA) followed by Bonferroni's post-test. The animal infection study analysis was performed using the nonparametric Mann-Whitney $U$-test. $P<0.05$ was considered statistically significant.

\section{RESULTS}

\section{Effect of Catecholamines on A. hydrophila Growth}

In this study, NE, Epi, Dopa, L-dopa, tyrosine, and tyramine were evaluated for A. hydrophila growth promotion. The data showed that in serum-SAPI minimul medium, NE, Epi, Dopa, and L-dopa significantly stimulated the growth of A. hydrophila (Figure 1), and resulted in a 1.81-, 1.72-, 1.75-, and 1.72-fold increase $(P<0.05)$ in the specific growth rate of $A$. hydrophila, respectively $(0.58 \pm 0.12,0.55 \pm 0.03,0.56 \pm 0.03$, and 0.55 $\pm 0.15 \mathrm{~h}^{-1}$ in the presence of NE, Epi, Dopa, and L-dopa,

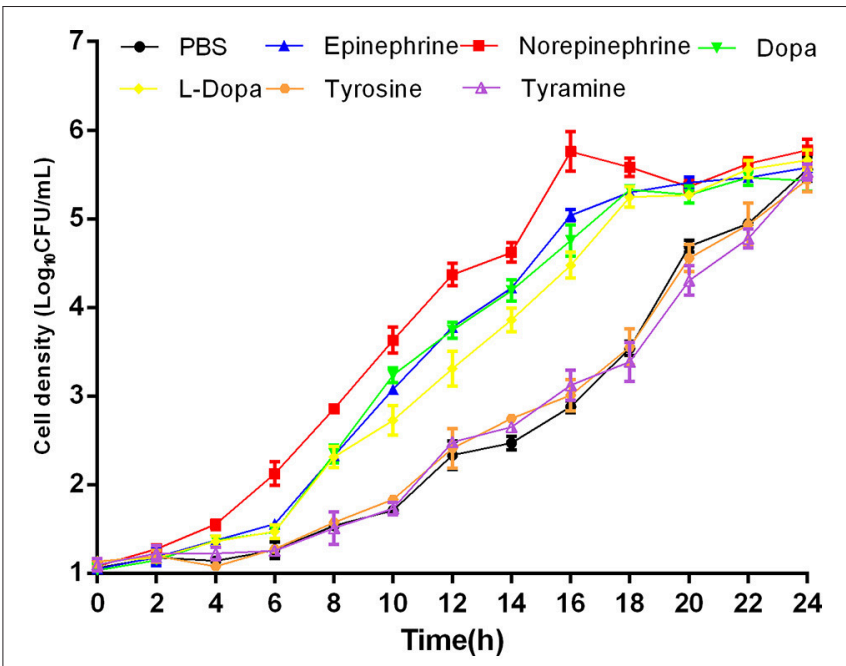

FIGURE 1 | Growth of $A$. hydrophila NJ-35 in the presence of NE and NE biosynthetic intermediates. An initial inoculum of $A$. hydrophila at approximately $10^{2} \mathrm{CFU} / \mathrm{ml}$ was grown in serum-containing medium supplemented with individual hormones at a concentration of $100 \mu \mathrm{M}$ at $28^{\circ} \mathrm{C}$ in a humid atmosphere containing $5 \% \mathrm{CO}_{2}$. Results are shown as the means \pm SEM from four independent replicates.

respectively, compared to $0.32 \pm 0.09 \mathrm{~h}^{-1}$ for the control). The growth of $A$. hydrophila in the presence of tyrosine and tyramine exhibited no increase compared to the control $(0.33 \pm 0.23$ and $0.30 \pm 0.29 \mathrm{~h}^{-1}$ in the presence of tyrosine and tyramine, respectively, compared to $0.32 \pm 0.09 \mathrm{~h}^{-1}$ for the control).

To evaluate whether growth promotion by catecholamines could be blocked, we performed growth inhibition tests employing adrenergic and dopaminergic-type receptor antagonists. The data demonstrated that the $\alpha$-adrenergic antagonist phentolamine was able to inhibit growth induction by Epi and NE but could not block growth responses to Dopa and L-dopa. The dopaminergic receptor antagonist chlorpromazine was only able to inhibit growth induction by Dopa (Figure 2). Furthermore, the $\beta$-adrenergic antagonist propranolol had no effect on NE-, Epi-, Dopa-, or L-dopa-induced growth. Receptor antagonists did not affect the growth of A. hydrophila when used alone (data not shown). This indicates that growth inhibition is the result of specific antagonism of the bacterial response to stress hormones but does not result from antagonist toxicity. To exclude the possibility that the receptor antagonists directly bind NE, we examined the iron release from transferrin in the presence of NE- phentolamine mixture by denaturing urea-PAGE analysis (Figure 7). We found that the addition of receptor antagonists did not influence the iron removal from transferrin by NE, indicating that the antagonist effect was caused by a blockade of bacteria to NE.

\section{Effects of Catecholamines on Biofilm Formation and Bacterial Adherence}

Crystal violet staining assays were carried out to examine whether catecholamines impacted the formation of biofilms. The results showed that biofilm formation was increased after $16 \mathrm{~h}$ by the 

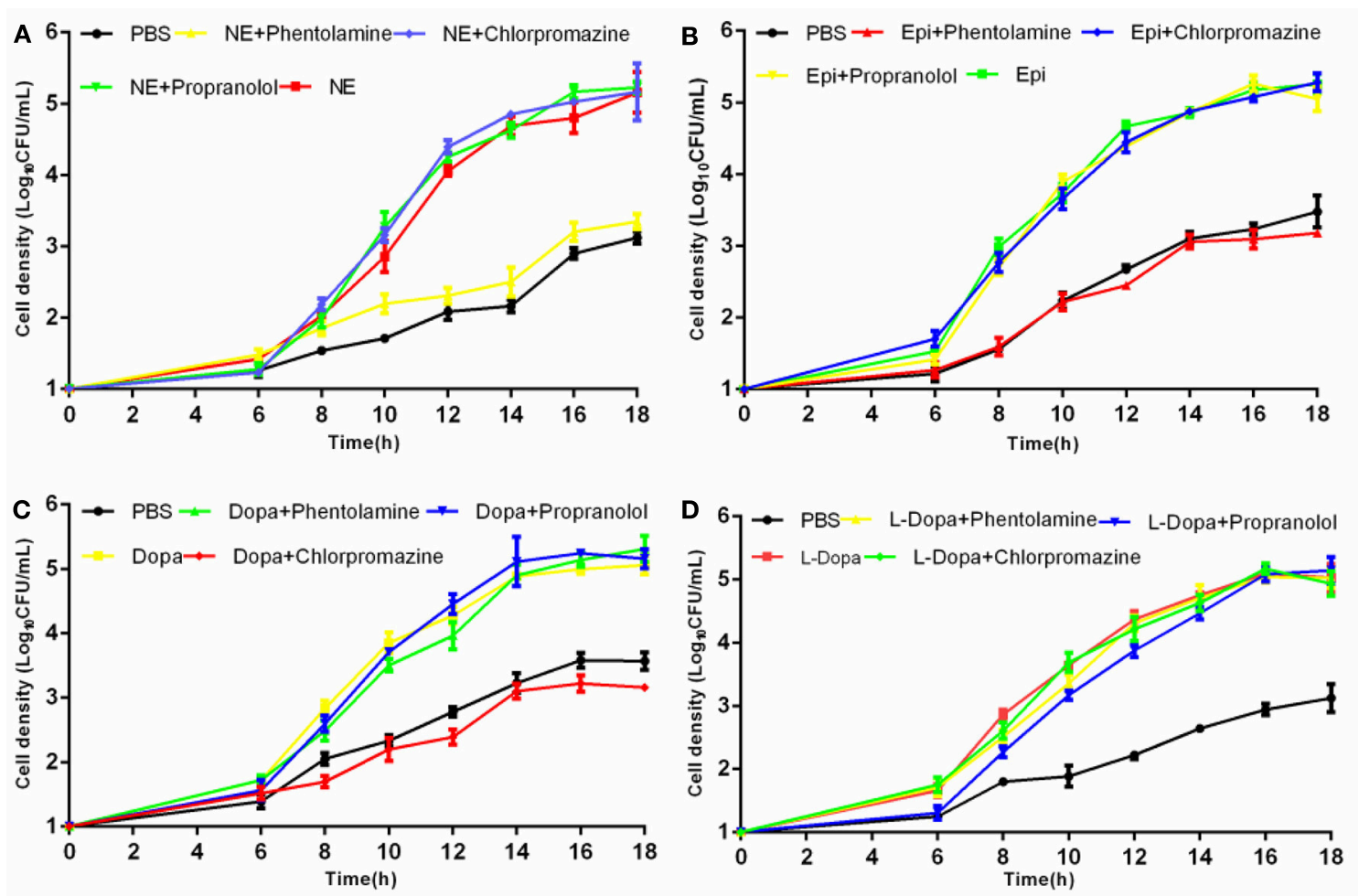

FIGURE 2 | Effects of receptor antagonists on hormone-induced growth responsiveness in A. hydrophila NJ-35. Catecholamine receptor antagonists were added at a concentration of $400 \mathrm{mM}$ to investigate the growth induced by NE (A), Epi (B), Dopa (C), and L-dopa (D). Results are shown as the means \pm SEM from three independent replicates.

addition of NE, Epi, or L-dopa, and NE exerted the strongest effect on biofilm formation (Figure 3). Before performing this assay, we examined the growth of $A$. hydrophila at an initial inoculum of $5 \times 10^{6} \mathrm{CFU} / \mathrm{ml}$. No significant modification in growth was observed following exposure to the hormones used in this assay (data not shown). This result indicates that the enhancement of biofilm formation is not attributable to the effects of the hormones on growth rate.

An in vitro adhesion assay showed that supplementation with $\mathrm{NE}$ and Dopa could significantly enhance A. hydrophila adhesion to HEp-2 cells (Figure 4). Other molecules used in this assay induced no changes in adherence. Our findings demonstrated that pathogenic phenotypes such as biofilm formation and adhesion to HEp-2 cells can be regulated by different stress hormones.

\section{NE Enhances the Systemic Spread of}

\section{A. hydrophila In vivo}

To corroborate whether NE can affect the course of A. hydrophila proliferation in vivo, we performed an infection assay involving the artificial modulation of catecholamine levels in mice. Groups of six mice were intragastrically administered 0 or $1 \mathrm{mg}$ of NE in $400 \mu \mathrm{l}$ of PBS $12 \mathrm{~h}$ before infection with A. hydrophila NJ-35. Mice were sacrificed at $6 \mathrm{~h}$ postinfection to collect their spleens and lungs, and the bacterial loads were assessed in these

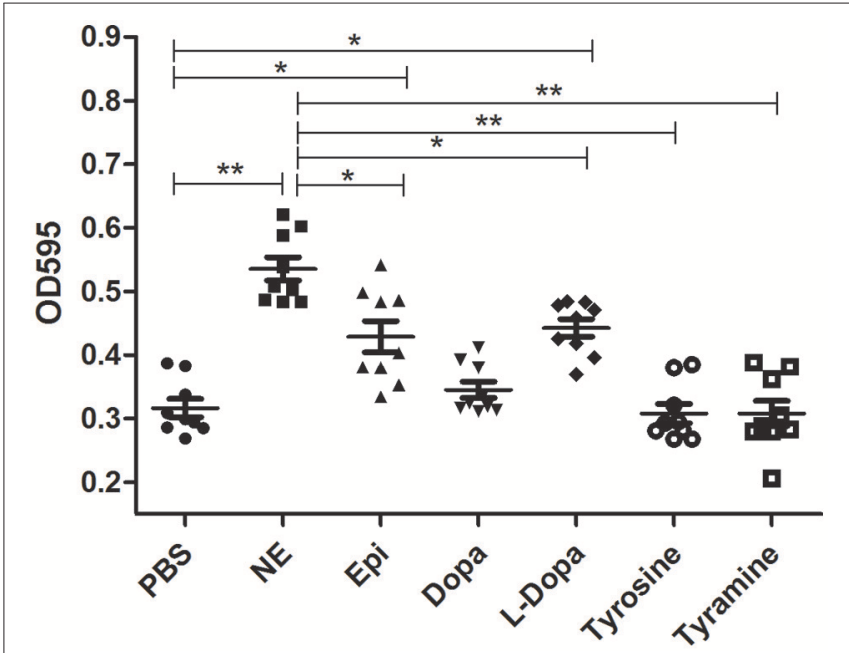

FIGURE 3 | Biofilm formation of $A$. hydrophila NJ-35 in the presence of NE and NE biosynthetic intermediates. $A$. hydrophila NJ-35 was grown in serum-containing medium supplemented with individual hormones at a concentration of $100 \mu \mathrm{M}$. Crystal violet staining was performed to examine biofilm formation. ${ }^{\star} P<0.05$ or ${ }^{\star *} P<0.01$.

tissues. The pre-treatment of mice with $\mathrm{NE}$ after challenge with A. hydrophila NJ-35 resulted in marked enhancements in bacterial colonization compared to non-pre-treated controls. 


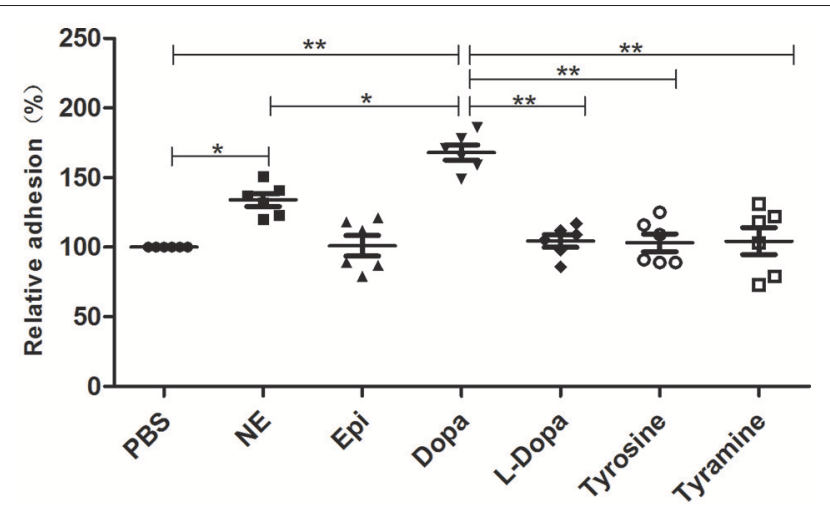

FIGURE 4 | A. hydrophila NJ-35 adhesion to HEp-2 cells in the presence of NE and NE biosynthetic intermediates. $A$. hydrophila NJ-35 was grown in serum-SAPI medium supplemented with individual hormones at a concentration of $100 \mu \mathrm{M}$ for $18 \mathrm{~h}$ at $28^{\circ} \mathrm{C}$ and was then seeded into HEp-2 cells at an $\mathrm{MOI}$ of 1 . Adherent bacterial numbers were counted by plating out the lysates of infected HEp-2 cells on LB agar. ${ }^{*} P<0.05$ or ${ }^{\star \star} P<0.01$.

As shown in Figure 5, approximate 3- and 2-fold increases in the spleen and lung, respectively, were observed compared to the controls. This result demonstrated that NE can increase the systemic spread of $A$. hydrophila NJ-35 and accelerate the course of infection.

\section{Involvement of Tf in Growth Stimulation by NE in SAPI Medium}

To evaluate the role of transferrin in the NE-induced growth stimulation of $A$. hydrophila, we performed a growth assay employing $39 \mu \mathrm{M}$ transferrin instead of serum in SAPI medium. As expected, NE enhanced the growth of A. hydrophila in SAPI medium containing holotransferrin (Figure 6). This result is similar to the results that we obtained in the NE-mediated growth stimulation assay with the addition of serum into SAPI medium (Figure 1). In contrast, $A$. hydrophila growth did not significantly change in apotransferrin-containing medium in the presence of NE. These results indicate that holotransferrin can take the place of serum in NE-induced growth stimulation. Further, the urea-PAGE analysis of the Tf-NE complex showed that in the presence of NE, purified diferric Tf was gradually converted into the monoferric or apo forms of Tf (Figure 7).

\section{Stimulation of $A$. hydrophila Growth Is Not Mediated by Amonabactin}

To determine whether NE-enhanced growth of $A$. hydrophila NJ35 was mediated by amonabactin, we constructed an isogenic deletion mutant of amoA. The CAS plate assay showed that the $\triangle a m o A$ mutant failed to produce amonabactin (Figure 8). The addition of $100 \mu \mathrm{M}$ NE enhanced the growth of the $\triangle a m o A$ mutant (Figure 9) and resulted in a 1.45-fold increase $(P<0.05)$ in the specific growth rate $\left(0.32 \pm 0.06 \mathrm{~h}^{-1}\right.$ in the presence of NE, compared to $0.22 \pm 0.03 \mathrm{~h}^{-1}$ for the untreated control), while the similar NE-mediated growth promotion was observed for the wild-type strain $\left(0.45 \pm 0.23 \mathrm{~h}^{-1}\right.$ in the

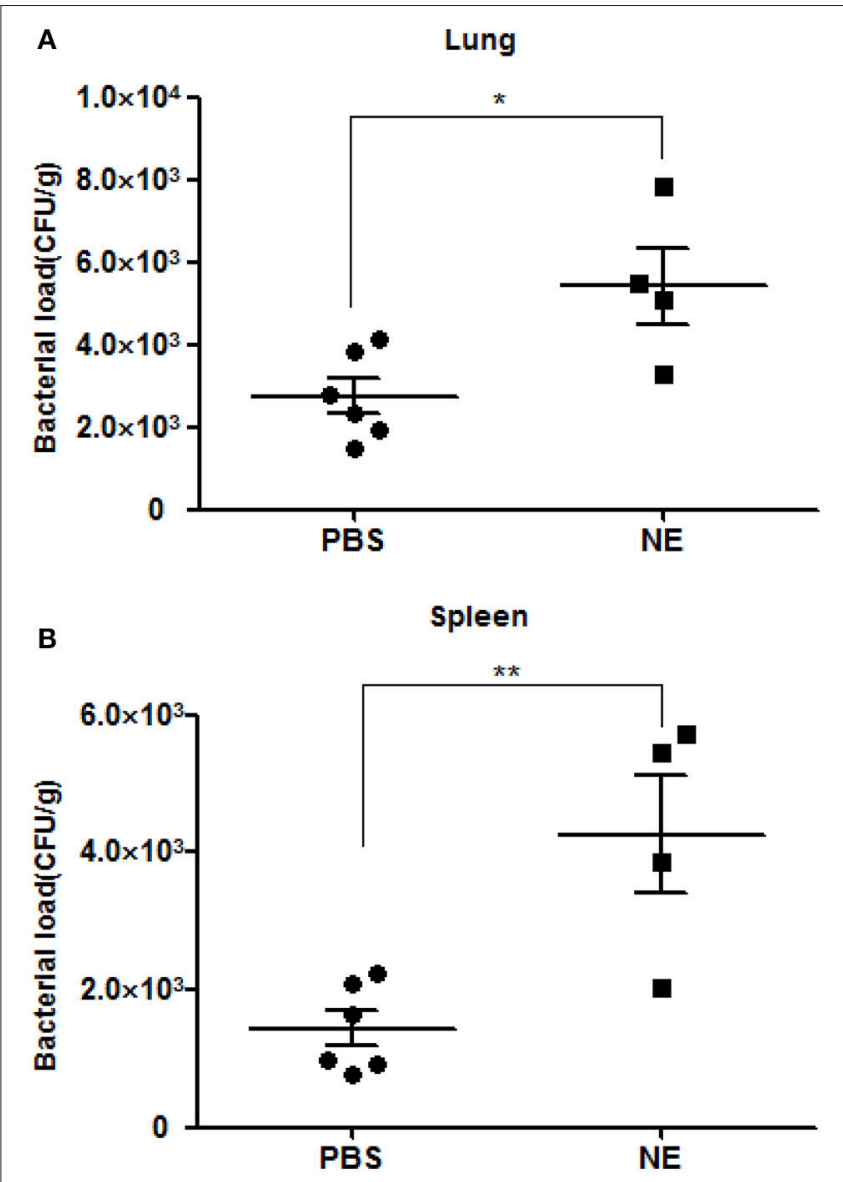

FIGURE 5 | Bacterial loads in mice with or without NE pre-treatment. Bacterial loads in the spleen (A) and lung (B) are expressed as CFU/g of tissue. The experiment was repeated three times. ${ }^{\star} P<0.05$ or ${ }^{\star \star} P<0.01$.

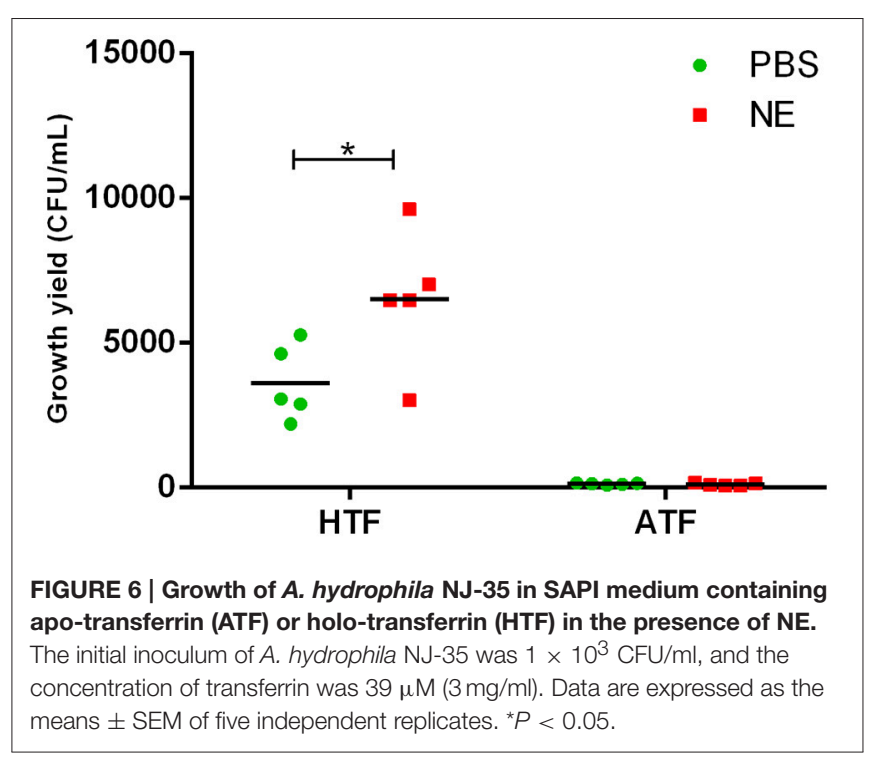




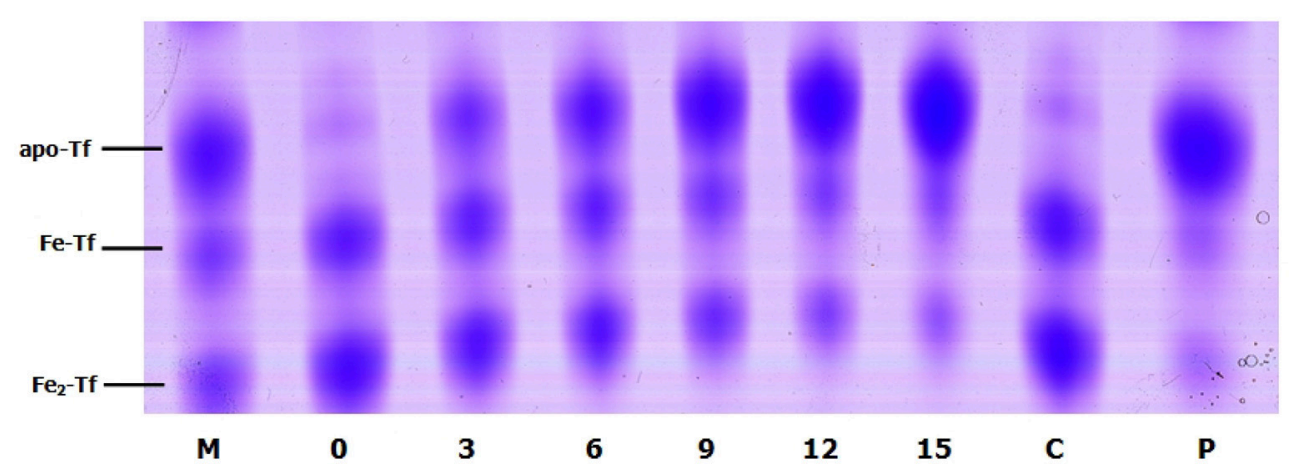

FIGURE 7 | Urea polyacrylamide gel electrophoresis demonstrating iron removal from Tf in the presence of NE over time. A. hydrophila NJ-35 was inoculated in HTF-SAPI medium containing $100 \mathrm{mM}$ Tris-HCl buffer at $37^{\circ} \mathrm{C}$. The numbers below the lanes represent the number of hours of incubation with $\mathrm{NE}$. Lane $\mathrm{M}$ contains the iron-free (apo-Tf), monoferric (Fe-Tf), and saturated ( $\mathrm{Fe}_{2}$-Tf) isoforms as markers. Lane $\mathrm{C}$ shows $\mathrm{Fe}_{2}$-Tf incubated in the medium for $15 \mathrm{~h}$ without $\mathrm{NE}$. Lane $\mathrm{P}$ shows $\mathrm{Fe}_{2}$-Tf incubated in the medium for $15 \mathrm{~h}$ with NE and phentolamine.

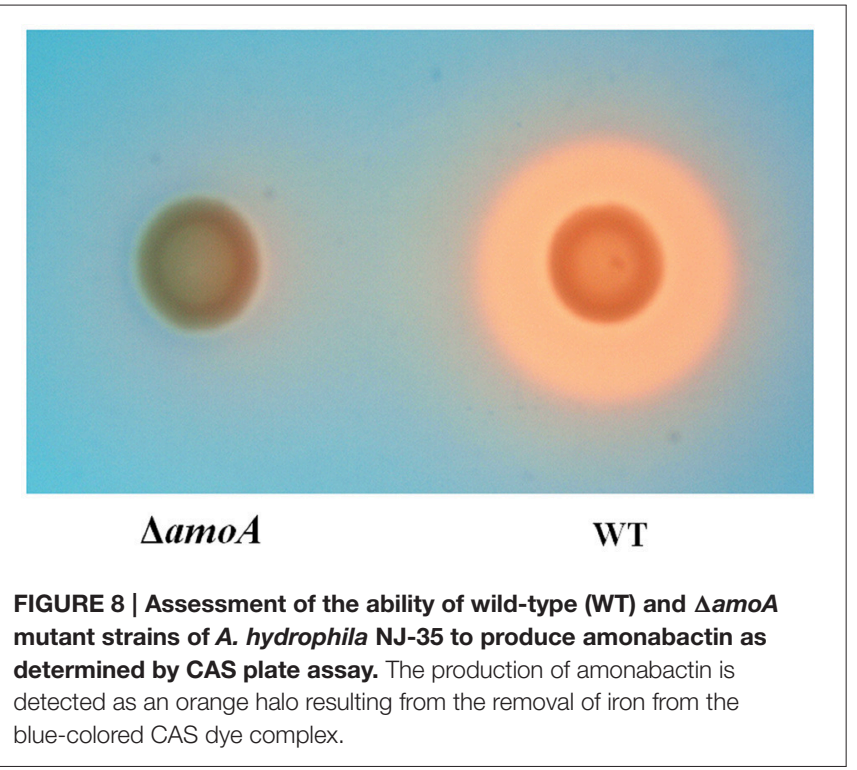

presence of NE, compared to $0.32 \pm 0.20 \mathrm{~h}^{-1}$ for the untreated control). Moreover, compared with the wild-type strain, the $\triangle a m o A$ mutant strain showed a significant growth defect in serum-SAPI medium (Figure 10A). The complementary strain, $\mathrm{C} \triangle a m o A$ could restore the production of amonabactin, and the $\mathrm{C} \triangle a m o A$ growth was almost restored to the wildtype level in the presence or absence of NE (data not shown). These data indicated that amonabactin plays a crucial role in growth in serum-based medium, whereas the ability of $A$. hydrophila to synthesize amonabactin is not essential for NE-dependent growth promotion in serum-supplemented medium.

\section{TonB2 Contributes to NE-Dependent Growth Promotion in A. hydrophila}

As the present study indicated that NE-enhanced A. hydrophila growth was attributable to increased iron availability in the bacterial cell, we determined whether this process required

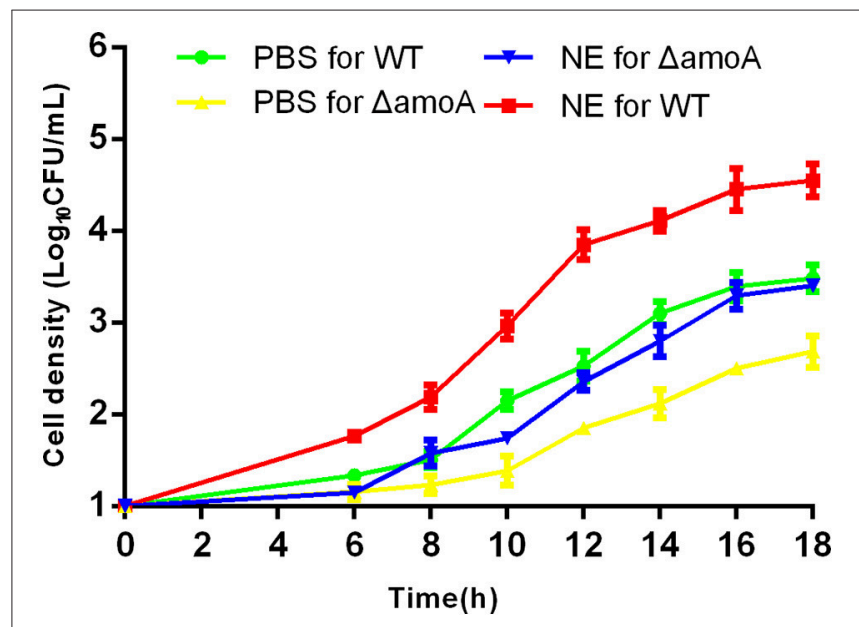

FIGURE 9 | Growth stimulation by NE is independent of amonabactin. A. hydrophila NJ-35 wild-type (WT) and $\triangle a m o A$ strains were grown in serum-supplemented SAPI medium in the presence or absence of NE. Results are shown as the means \pm SEM from four independent replicates.

the TonB-dependent transport system. The TonB system is responsible for energizing transporters in the outer membrane (Postle and Larsen, 2007). A homology search of the genome sequence of $A$. hydrophila NJ-35 revealed the presence of three TonB systems, encoded by tonB1-exbB1-exbD1, tonB2-2-exbB2exbD2-tonB2-1, and tonB3-exbB3-exbB3-exbD3 (Figure 10B). To determine which TonB system is involved in the NE-mediated growth enhancement of $A$. hydrophila, we constructed tonB1, tonB2-1, tonB2-2, and tonB3 single deletion mutants. The $\triangle$ tonB2-1 and $\triangle$ tonB2-2 strains demonstrated no responses to $\mathrm{NE}$ in serum-containing medium $(0.13 \pm 0.09$ and 0.13 $\pm 0.04 \mathrm{~h}^{-1}$ in the presence of NE, respectively, compared to $0.12 \pm 0.03$ and $0.11 \pm 0.05 \mathrm{~h}^{-1}$ for the untreated control), whereas the growth of the complemented strain was almost restored to the level of the wild-type strain in responses to $\mathrm{NE}$ (Figure 11). However, NE still enhanced the growth of both the $\triangle$ tonB1 and $\triangle$ tonB3 mutants (Figure 11) and resulted in a 2.0- 


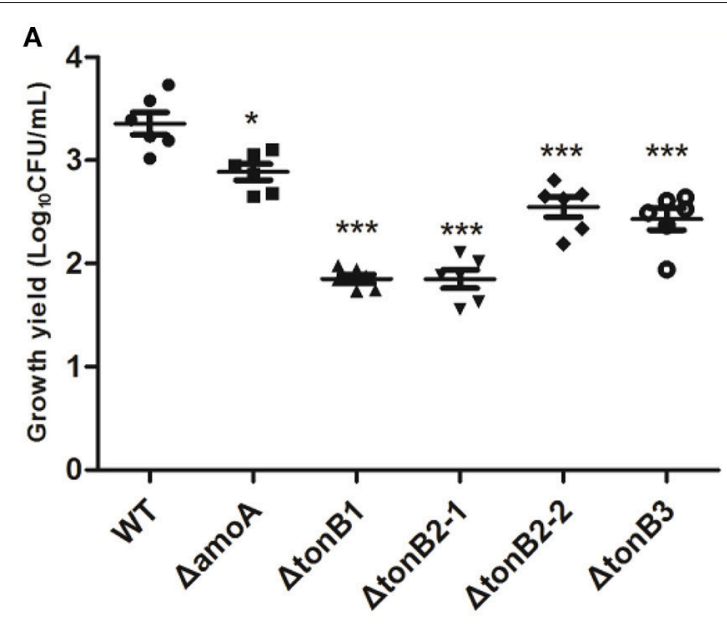

B

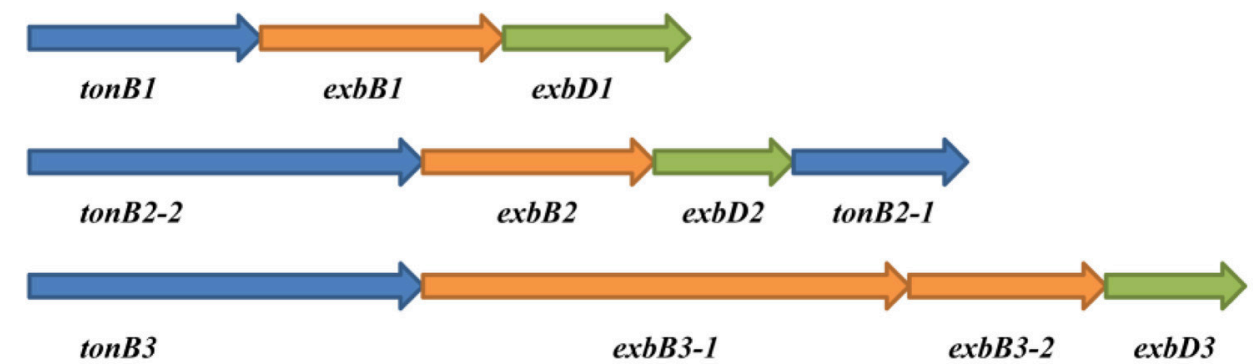

FIGURE 10 | Growth of the wild-type A. hydrophila strain, its derivative amoA and the tonB mutants in serum-supplemented medium (A) and genetic organization of the three-TonB system gene clusters of $A$. Hydrophila NJ-35 (B). The growth yield is expressed as Log ${ }_{10}$ CFU/ml, and results are shown as the means \pm SEM from six independent replicates. ${ }^{\star} P<0.05$, or ${ }^{\star \star *} P<0.001$.

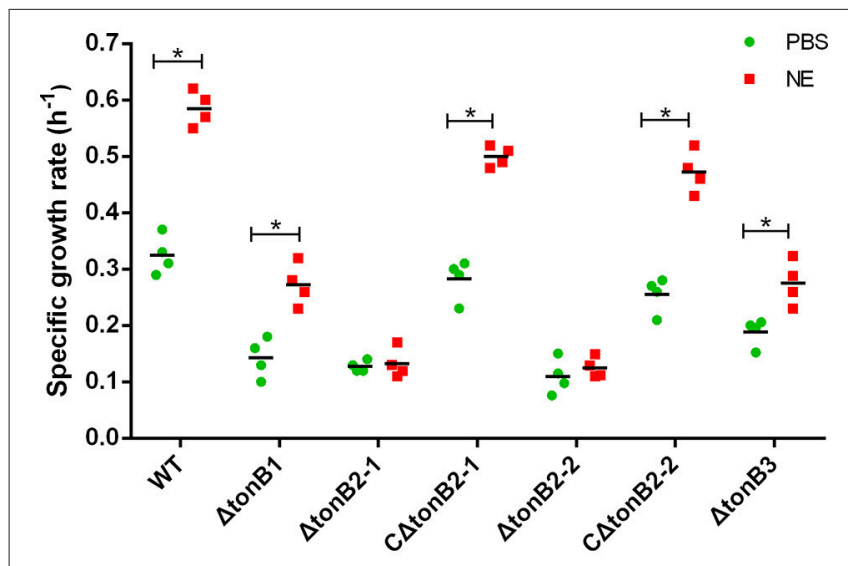

FIGURE 11 | TonB2-dependent growth stimulation by NE in serum-supplemented SAPI medium. The specific growth rate calculated from the cell densities measured during exponential growth phase. The results are shown as the means \pm SEM from four independent replicates. ${ }^{*} P<0.05$.

and 1.5 -fold increase $(P<0.05)$ in the specific growth rate, respectively $\left(0.28 \pm 0.07\right.$ and $0.28 \pm 0.06 \mathrm{~h}^{-1}$ in the presence of $\mathrm{NE}$, respectively, compared to $0.14 \pm 0.03$ and $0.19 \pm 0.10 \mathrm{~h}^{-1}$ for the untreated control). In addition, the growth yields of all four mutants were markedly lower than that of the parental strain in serum-supplemented medium (Figure 10A). These results indicated that only the TonB2 system is necessary for NEdependent growth stimulation in serum-containing medium. Furthermore, it is notable that all three TonB systems are required for the optimal growth of $A$. hydrophila in serumcontaining medium.

\section{DISCUSSION}

In the recent years, microbial endocrinology has revealed that many microorganisms have evolved specific mechanisms to sense and respond to stress hormones. However, most investigations to date have concentrated on mammalian pathogens. Epinephrine, dopamine and norepinephrine are the predominant stress hormones that are released from the chromaffin tissue located in the head kidney (Perry and Capaldo, 2011) as well as from adrenergic nerve endings in fish (Gamperl et al., 1994). To determine the effect of the catecholamine stress hormones on A. hydrophila growth, in this study, we used a minimal medium (SAPI) supplemented with serum to simulate in vivo iron-restricted environment. In considering the catecholamine concentrations used in the present study, it should be emphasized that micromolar concentrations are meant to mimic the concentrations in the host. However, because catecholamines levels can change according to stress level, tissue location and 
circadian rhythm, the actual catecholamine concentrations inside tissues and blood are difficult to accurately determine (Li et al., 2012). For example, higher concentrations can be found in innervated tissues such as the gut, and the concentration of $\mathrm{NE}$ can be up to $10 \mathrm{mM}$ in neuronal synapses (Lyte, 2004). The concentration of catecholamines used in this study (100 $\mu \mathrm{M})$ is, therefore, biologically relevant. Similar concentration range has been used in a previous study, which has investigated A. hydrophila responses to catecholamines (Kinney et al., 1999).

Our study demonstrated that NE, Epi, Dopa, and Ldopa could significantly increase $A$. hydrophila growth in SAPI-serum medium, whereas tyrosine and tyramine did not significantly alter the bacterial growth. These results suggest that A. hydrophila has evolved catecholamine response systems for the specific hormones. Further investigation demonstrated that the induced growth of $A$. hydrophila by Epi and NE could be blocked by $\alpha$ - but not $\beta$-receptor antagonists; however, the $\alpha$ receptor antagonist did not neutralize induction by Dopa. In contrast, the dopaminergic receptor antagonist blocked growth induction by Dopa but did not demonstrate any effects on other catecholamines with the ability to stimulate bacterial growth. This may reflect the fact that bacterial receptor systems for catecholamine possess a degree of specificity, similar to mammalian catecholamine receptors (Yang et al., 2014). In terms of bacterial catecholamine receptors, there is so far no genomic evidence for the existence of a classical adrenergic receptor motif in bacterial species. However, Clarke et al. (2006) reported that $\mathrm{NE}$ was able to bind to the E. coli O157:H7 two-component regulator sensor kinase QseC, leading to the hypothesis that QseC is the bacterial catecholamine receptor. Later on, there are increasing reports of alternative receptors which participate in bacterial responsiveness to adrenaline or noradrenaline, such as BasSR and CpxAR two-component signal transduction systems (Humphreys et al., 2004; Marchal et al., 2004). In this paper, the evidence from the antagonist experiments indicates the presence of specific recognition systems for NE, Epi and Dop that are essential for induction of bacterial growth. However, unequivocal evidence for the existence of bacterial $\alpha$-adrenergic and dopaminergic receptors in A. hydrophila will require further investigation.

In this study, we showed that catecholamines can increase biofilm formation and adhesion, which are crucial for A. hydrophila infections. Norepinephrine exerted the strongest impact on biofilm formation, but the effects of Dopa on bacterial adhesion were greater than those of norepinephrine. These discrepancies may be attributable to the possibility that there is more than one system to respond to stress hormones. In E. coli O157:H7, neuroendocrine hormones are sensed by the QseBC and QseEF two component systems (Rasko et al., 2008), which are involved in regulating motility and the expression of the enterocyte effacement (LEE) gene locus, respectively (Reading et al., 2007). In this regard, it may be interesting to further evaluate which systems contribute to catecholamine hormone sensing and the regulation of stress-associated gene expression in A. hydrophila.

Additionally, in vivo experiment showed that $\mathrm{NE}$ administration increased bacterial loads in the spleen and lung of mice at $6 \mathrm{~h}$ post-infection. It is not clear which mechanism encourages the colonization in tissues by $A$. hydrophila. However, the addition of NE resulted in enhanced bacterial adherence and biofilm production in vitro, which implies that NE increased fitness for transmission of A. hydrophila in mice. Furthermore, whether NE impairs host immune system is not known at this time, but is a future investigative objective of our laboratories.

Intriguingly, we found that all growth-stimulatory hormones employed in this assay contained a 3,4-dihydroxybenzoyl moiety. A previous study showed that the 3,4-dihydroxybenzoyl (catechol) structure is an essential element for the removal of iron from transferrin (Eisenhofer et al., 1996). Furthermore, some metabolites of catecholamine hormones that contain the catechol moiety, such as dihydroxyphenyl glycol, and dihydroxymandelic acid, have been demonstrated to exert growth-promoting effects (Nakano et al., 2007). Thus, we speculate that hormone-mediated growth promotion may be involved in facilitating the utilization of iron by bacteria.

In the host, the concentration of freely available iron is very low in the circulation. Ferric iron is generally sequestered by high-affinity iron-chelating proteins such as transferrin in the plasma (Schade and Caroline, 1946). Here, we investigated the role of transferrin in the NE-induced A. hydrophila growth. Similar to the effect of serum, the addition of transferrin to SAPI medium enhanced the growth of $A$. hydrophila. Further, we demonstrated that iron could be released from transferrin in the presence of $\mathrm{NE}$, as determined by denaturing urea-PAGE analysis. The data indicated that $\mathrm{NE}$ has the ability to remove iron from ferric transferrin in A. hydrophila.

For many pathogens, the acquisition of iron generally involves the production and extraction of siderophores (Meyer et al., 1996). Siderophores can capture iron from transferrin or inaccessible ferric iron found in the environment and subsequently deliver it into the bacterial cell through specialized uptake mechanisms (Schalk et al., 2012). A report from Barghouthi et al. (1989) showed that amonabactin is necessary for iron acquisition from Fe-transferrin in A. hydrophila. Further study showed that amoA was the key gene in the sevengene cluster involved in the biosynthesis of amonabactin in A. hydrophila 495A2 (Barghouthi et al., 1991). To determine whether amonabactin was required for NE-induced growth promotion of $A$. hydrophila NJ-35 in the iron-restricted medium, we inactivated the amoA gene. The data here showed that whether in the presence or absence of NE, the amoA mutant showed a significant reduction in growth when compared with wildtype, indicating that amonabactin plays a crucial role in bacterial growth in the restrictive environment of serumSAPI medium. However, the addition of NE significantly stimulated the growth of both wild-type and amoA mutant strains, suggesting that the NE-mediated iron acquisition from transferrin in iron-restricted medium is independent of the ability to synthesize amonabactin. The precise mechanism(s) by which NE modulates iron uptake from iron-Tf remain to be determined. Although, we speculate that NE-mediated iron uptake from iron-Tf might be due to NE acting directly in a siderophore-like manner, we could not exclude the possibility 
that NE-Tf interactions were releasing iron for subsequent uptake by bacterial iron acquisition systems. Future work will be carried out to elucidate this.

The process of siderophore-mediated iron acquisition from transferrin is energy-dependent. The active transport of ironsiderophore compounds across the outer membrane is energized by a complex of proteins called the TonB energy transduction system (Postle and Larsen, 2007). A. hydrophila possesses three TonB systems, similar to Vibrio vulnificus (Kustusch et al., 2012). To discover which TonB system was required in NE-mediated iron acquisition of $A$. hydrophila, we individually inactivated the three TonB systems. We found that all three ton $B$ mutants exhibited lower growth yields compared with the wild-type strain in SAPI medium containing serum, indicating that all three TonB systems were involved in iron acquisition from Fe-transferrin in serum. However, in the presence of NE, the tonB2 mutant demonstrated no growth promotion, although this stimulatory effect was observed for both the tonB1 and tonB3 mutants. This result indicates that the TonB2 system may play an important role in NE-induced iron acquisition from Fe-transferrin in serum, and TonB2-dependent growth promotion might be important for A. hydrophila infection in the host under stress. Moreover, the findings showed that both the $\Delta$ tonB2-1 and $\triangle$ tonB2-2 strains could not respond to NE in serum-containing medium, indicating that TonB2-1 and TonB22 are absolutely required for the function of the TonB2 system in A. hydrophila. The observation that NE-mediated growth promotion of $A$. hydrophila is TonB-dependent strongly suggests that a specific TonB-dependent outer membrane receptor might be involved in the transport of iron from transferrin via norepinephrine. Further studies are necessary to demonstrate which receptors are needed for NE-induced growth promotion of A. hydrophila.

\section{REFERENCES}

Abolghait, S. K. (2013). Suicide plasmid-dependent IS $_{1}$-element untargeted integration into Aeromonas veronii bv. sobria generates brown pigmentproducing and spontaneous pelleting mutant. Curr. Microbiol. 67, 91-99. doi: 10.1007/s00284-013-0335-4

Bansal, T., Englert, D., Lee, J., Hegde, M., Wood, T. K., and Jayaraman, A. (2007). Differential effects of epinephrine, norepinephrine, and indole on Escherichia coli O157:H7 chemotaxis, colonization, and gene expression. Infect. Immun. 75, 4597-4607. doi: 10.1128/IAI.00630-07

Barghouthi, S., Payne, S. M., Arceneaux, J. E., and Byers, B. R. (1991). Cloning, mutagenesis, and nucleotide sequence of a siderophore biosynthetic gene (amoA) from Aeromonas hydrophila. J. Bacteriol. 173, 5121-5128. doi: 10.1128/jb.173.16.5121-5128.1991

Barghouthi, S., Young, R., Olson, M. O., Arceneaux, J. E., Clem, L. W., and Byers, B. R. (1989). Amonabactin, a novel tryptophan- or phenylalanine-containing phenolate siderophore in Aeromonas hydrophila. J. Bacteriol. 171, 1811-1816. doi: 10.1128/jb.171.4.1811-1816.1989

Burton, C. L., Chhabra, S. R., Swift, S., Baldwin, T. J., Withers, H., Hill, S. J., et al. (2002). The growth response of Escherichia coli to neurotransmitters and related catecholamine drugs requires a functional enterobactin biosynthesis and uptake system. Infect. Immun. 70, 5913-5923. doi: 10.1128/iai.70.11.5913-5923.2002

Clarke, M. B., Hughes, D. T., Zhu, C., Boedeker, E. C., and Sperandio, V. (2006). The QseC sensor kinase: a bacterial adrenergic receptor. Proc. Natl. Acad. Sci. U.S.A. 103, 10420-10425. doi: 10.1073/pnas.0604343103
In conclusion, our results demonstrate that the stress hormones NE, Epi, and Dopa stimulate bacterial growth and increase biofilm formation and cell adhesion ability in $A$. hydrophila. These results partially explain why stress increases the risk of $A$. hydrophila infection in fish. We also provide evidence to suggest that NE-induced growth promotion correlates with iron uptake from transferrin facilitated by norepinephrine. Although, the mechanisms of communication between bacteria and their hosts are not well understood, the modulation of bacterial growth and virulence by stress hormones brings a new perspective to infectious disease processes induced by bacteria.

\section{AUTHOR CONTRIBUTIONS}

YL and YD conceived the study and drafted the paper. YD and JL performed most of the experiments described in the manuscript. $\mathrm{HD}, \mathrm{NW}$, and FA helped with the experiments. CL provided valuable suggestions of the manuscript. All authors read and approved the final manuscript.

\section{ACKNOWLEDGMENTS}

This work was supported by the National Nature Science Foundation of China (31372454), Aquatic Three New Projects in Jiangsu Province (D2015-13), and Priority Academic Program Development of Jiangsu Higher Education Institutions (PAPD).

\section{SUPPLEMENTARY MATERIAL}

The Supplementary Material for this article can be found online at: http://journal.frontiersin.org/article/10.3389/fcimb. 2016.00183/full\#supplementary-material

Eisenhofer, G., Aneman, A., Hooper, D., Rundqvist, B., and Friberg, P. (1996). Mesenteric organ production, hepatic metabolism, and renal elimination of norepinephrine and its metabolites in humans. J. Neurochem. 66, 1565-1573. doi: 10.1046/j.1471-4159.1996.66041565.x

Freestone, P. P., Haigh, R. D., and Lyte, M. (2007). Blockade of catecholamineinduced growth by adrenergic and dopaminergic receptor antagonists in Escherichia coli O157:H7, Salmonella enterica and Yersinia enterocolitica. BMC Microbiol. 7:8. doi: 10.1186/1471-2180-7-8

Freestone, P. P., and Lyte, M. (2008). Microbial endocrinology: experimental design issues in the study of interkingdom signalling in infectious disease. $A d v$. Appl. Microbiol. 64, 75-105. doi: 10.1016/S0065-2164(08)00402-4

Freestone, P. P., Haigh, R. D., Williams, P. H., and Lyte, M. (2003). Involvement of enterobactin in norepinephrine-mediated iron supply from transferrin to enterohaemorrhagic Escherichia coli. FEMS Microbiol. Lett. 222, 39-43. doi: 10.1016/S0378-1097(03)00243-X

Funahashi, T., Tanabe, T., Miyamoto, K., Tsujibo, H., Maki, J., and Yamamoto, S. (2013). Characterization of a gene encoding the outer membrane receptor for ferric enterobactin in Aeromonas hydrophila ATCC 7966(T). Biosci. Biotechnol. Biochem. 77, 353-360. doi: 10.1271/bbb.120774

Galindo, C. L., Gutierrez, C. Jr., and Chopra, A. K. (2006). Potential involvement of galectin-3 and SNAP23 in Aeromonas hydrophila cytotoxic enterotoxin-induced host cell apoptosis. Microb. Pathog. 40, 56-68. doi: 10.1016/j.micpath.2005.11.001

Gamperl, A. K., Vijayan, M. M., and Boutilier, R. G. (1994). Experimental control of stress hormone levels in fishes: techniques and applications. Rev. Fish Biol. Fish. 4, 215-255. doi: 10.1007/BF00044129 
Humphreys, S., Rowley, G., Stevenson, A., Anjum, M. F., Woodward, M. J., Gilbert, S., et al. (2004). Role of the two-component regulator CpxAR in the virulence of Salmonella enterica serotype Typhimurium. Infect. Immun. 72, 4654-4661. doi: 10.1128/IAI.72.8.4654-4661.2004

Kasvosve, I., and Delanghe, J. (2002). Total iron binding capacity and transferrin concentration in the assessment of iron status. Clin. Chem. Lab. Med. 40, 1014-1018. doi: 10.1515/CCLM.2002.176

Kinney, K. S., Austin, C. E., Morton, D. S., and Sonnenfeld, G. (1999). Catecholamine enhancement of Aeromonas hydrophila growth. Microb. Pathog. 26, 85-91. doi: 10.1006/mpat.1998.0251

Kinney, K. S., Austin, C. E., Morton, D. S., and Sonnenfeld, G. (2000). Norepinephrine as a growth stimulating factor in bacteria-mechanistic studies. Life Sci. 67, 3075-3085. doi: 10.1016/S0024-3205(00)00891-2

Kustusch, R. J., Kuehl, C. J., and Crosa, J. H. (2012). The $t$ tpC gene is contained in two of three TonB systems in the human pathogen Vibrio vulnificus, but only one is active in iron transport and virulence. J. Bacteriol. 194, 3250-3259. doi: 10.1128/JB.00155-12

Lacoste, A., Jalabert, F., Malham, S. K., Cueff, A., and Poulet, S. A. (2001). Stress and stress-induced neuroendocrine changes increase the susceptibility of juvenile oysters (Crassostrea gigas) to Vibrio splendidus. Appl. Environ. Microbiol. 67, 2304-2309. doi: 10.1128/AEM.67.5.2304-2309.2001

Li, L., Xu, Z., Zhou, Y., Sun, L., Liu, Z., Chen, H., et al. (2012). Global effects of catecholamines on Actinobacillus pleuropneumoniae gene expression. PloS ONE 7:e31121. doi: 10.1371/journal.pone.0031121

Lindqvist, R., and Barmark, G. (2014). Specific growth rate determines the sensitivity of Escherichia coli to lactic acid stress: implications for predictive microbiology. Biomed. Res. Int. 2014:471317. doi: 10.1155/2014/471317

Lyte, M. (2004). Microbial endocrinology and infectious disease in the 21st century. Trends Microbiol. 12, 14-20. doi: 10.1016/j.tim.2003.11.004

Lyte, M., Arulanandam, B. P., and Frank, C. D. (1996). Production of Shiga-like toxins by Escherichia coli O157:H7 can be influenced by the neuroendocrine hormone norepinephrine. J. Lab. Clin. Med. 128, 392-398. doi: 10.1016/S0022-2143(96)80011-4

Maltz, M., LeVarge, B. L., and Graf, J. (2015). Identification of iron and heme utilization genes in Aeromonas and their role in the colonization of the leech digestive tract. Front. Microbiol. 6:763. doi: 10.3389/fmicb.2015.00763

Marchal, K., De Keersmaecker, S., Monsieurs, P., van Boxel, N., Lemmens, K., Thijs, G., et al. (2004). In silico identification and experimental validation of PmrAB targets in Salmonella typhimurium by regulatory motif detection. Genome Biol. 5:R9. doi: 10.1186/gb-2004-5-2-r9

Melton-Witt, J. A., McKay, S. L., and Portnoy, D. A. (2012). Development of a single-gene, signature-tag-based approach in combination with alanine mutagenesis to identify listeriolysin $\mathrm{O}$ residues critical for the in vivo survival of Listeria monocytogenes. Infect. Immun. 80, 2221-2230. doi: 10.1128/IAI.06196-11

Meyer, J. M., Neely, A., Stintzi, A., Georges, C., and Holder, I. A. (1996). Pyoverdin is essential for virulence of Pseudomonas aeruginosa. Infect. Immun. $64,518-523$.

Morales, V. M., Backman, A., and Bagdasarian, M. (1991). A series of wide-hostrange low-copy-number vectors that allow direct screening for recombinants. Gene 97, 39-47. doi: 10.1016/0378-1119(91)90007-X

Nakano, M., Takahashi, A., Sakai, Y., Kawano, M., Harada, N., Mawatari, K., et al. (2007). Catecholamine-induced stimulation of growth in Vibrio species. Lett. Appl. Microbiol. 44, 649-653. doi: 10.1111/j.1472-765X.2007.02136.x

Pang, M., Jiang, J., Xie, X., Wu, Y., Dong, Y., Kwok, A. H., et al. (2015). Novel insights into the pathogenicity of epidemic Aeromonas hydrophila ST251 clones from comparative genomics. Sci. Rep. 5:9833. doi: 10.1038/srep09833

Parker, J. L., and Shaw, J. G. (2011). Aeromonas spp. clinical microbiology and disease. J. Infect. 62, 109-118. doi: 10.1016/j.jinf.2010.12.003

Perry, S. F., and Capaldo, A. (2011). The autonomic nervous system and chromaffin tissue: neuroendocrine regulation of catecholamine secretion in non-mammalian vertebrates. Auton. Neurosci. 165, 54-66. doi: 10.1016/j.autneu.2010.04.006
Peters, G., Faisal, M., Lang, T., and Ahmed, I. (1988). Stress caused by social interaction and its effect on susceptibility to Aeromonas hydrophila infection in rainbow trout Salmo gairdneri. Dis. Aquat. Organ. 4, 83-89. doi: $10.3354 /$ dao004083

Postle, K., and Larsen, R. A. (2007). TonB-dependent energy transduction between outer and cytoplasmic membranes. Biometals 20, 453-465. doi: 10.1007/s10534-006-9071-6

Rasko, D. A., Moreira, C. G., Li de, R., Reading, N. C., Ritchie, J. M., Waldor, M. K., et al. (2008). Targeting QseC signaling and virulence for antibiotic development. Science 321, 1078-1080. doi: 10.1126/science.1160354

Reading, N. C., Torres, A. G., Kendall, M. M., Hughes, D. T., Yamamoto, K., and Sperandio, V. (2007). A novel two-component signaling system that activates transcription of an enterohemorrhagic Escherichia coli effector involved in remodeling of host actin. J. Bacteriol. 189, 2468-2476. doi: 10.1128/JB.01848-06

Sandrini, S., Alghofaili, F., Freestone, P., and Yesilkaya, H. (2014). Host stress hormone norepinephrine stimulates pneumococcal growth, biofilm formation and virulence gene expression. BMC Microbiol. 14:180. doi: 10.1186/1471-2180-14-180

Schade, A. L., and Caroline, L. (1946). An iron-binding component in human blood plasma. Science 104:340. doi: 10.1126/science.104.2702.340

Schalk, I. J., Mislin, G. L., and Brillet, K. (2012). Structure, function and binding selectivity and stereoselectivity of siderophore-iron outer membrane transporters. Curr. Top. Membr. 69, 37-66. doi: 10.1016/B978-0-12-394390-3.00002-1

Schwyn, B., and Neilands, J. B. (1987). Universal chemical assay for the detection and determination of siderophores. Anal. Biochem. 160, 47-56. doi: 10.1016/0003-2697(87)90612-9

Sperandio, V., Torres, A. G., Jarvis, B., Nataro, J. P., and Kaper, J. B. (2003). Bacteria-host communication: the language of hormones. Proc. Natl. Acad. Sci. U.S.A. 100, 8951-8956. doi: 10.1073/pnas.1537100100

Stepanovic, S., Vukovic, D., Dakic, I., Savic, B., and Svabic-Vlahovic, M. (2000). A modified microtiter-plate test for quantification of staphylococcal biofilm formation. J. Microbiol. Methods 40, 175-179. doi: 10.1016/s0167-7012(00)00122-6

Tenenbaum, T., Bloier, C., Adam, R., Reinscheid, D. J., and Schroten, H. (2005). Adherence to and invasion of human brain microvascular endothelial cells are promoted by fibrinogen-binding protein FbsA of Streptococcus agalactiae. Infect. Immun. 73, 4404-4409. doi: 10.1128/IAI.73.7.4404-4409.2005

Van Weerd, J. H., and Komen, J. (1998). The effects of chronic stress on growth in fish: a critical appraisal. Comp. Biochem. Physiol. A Mol. Integr. Physiol. 120, 107-112. doi: 10.1016/S1095-6433(98)10017-X

Wolz, C., Hohloch, K., Ocaktan, A., Poole, K., Evans, R. W., Rochel, N., et al. (1994). Iron release from transferrin by pyoverdin and elastase from Pseudomonas aeruginosa. Infect. Immun. 62, 4021-4027.

Yang, Q., Anh, N. D., Bossier, P., and Defoirdt, T. (2014). Norepinephrine and dopamine increase motility, biofilm formation, and virulence of Vibrio harveyi. Front. Microbiol. 5:584. doi: 10.3389/fmicb.2014.00584

Yao, Z., Wang, Z., Sun, L., Li, W., Shi, Y., Lin, L., et al. (2016). Quantitative proteomic analysis of cell envelope preparations under iron starvation stress in Aeromonas hydrophila. BMC Microbiol. 16:161. doi: 10.1186/s12866-016-0769-5

Conflict of Interest Statement: The authors declare that the research was conducted in the absence of any commercial or financial relationships that could be construed as a potential conflict of interest.

Copyright (๑) 2016 Dong, Liu, Pang, Du, Wang, Awan, Lu and Liu. This is an openaccess article distributed under the terms of the Creative Commons Attribution License (CC BY). The use, distribution or reproduction in other forums is permitted, provided the original author(s) or licensor are credited and that the original publication in this journal is cited, in accordance with accepted academic practice. No use, distribution or reproduction is permitted which does not comply with these terms. 